Journal of Mathematics and Statistics 8 (2): 248-252, 2012

ISSN 1549-3644

(C) 2012 Science Publications

\title{
Application of Optimal Control to the Epidemiology of Fowl Pox Transmission Dynamics in Poultry
}

\author{
Udofia Ekere Sunday and Inyama Simeon Chioma \\ Department of Mathematics and Computer Science, \\ School of Science, Federal University of Technology Owerri, Imo State, Nigeria
}

\begin{abstract}
Problem statement: In this study, we present the mathematical model of the transmission dynamics of fowl pox infection in poultry. Approach: It describes the interaction between the susceptible and the infected birds which results in a system of ordinary differential equation. Introducing the control which represents the effort in applying chemoprophylaxis control $u_{1}$ and treatment control $\mathrm{u}_{2}$ in birds with fowl pox, the system becomes a system of ordinary differential equations with control. Results: Our optimal control problem involves that in which the number of birds with latent and active fowl pox infections and the cost of treatment controls $u_{1}(t)$ and $u_{2}(t)$ were minimized subject to the differential Eq. 5-8. This involves the number of birds with active and latent fowl pox respectively as well as the cost of applying chemoprophylaxis control $\mathrm{u}_{1}$ and treatment $\mathrm{u}_{2}$ in birds with fowl pox. Conclusion: Analysing the model using Pontryagin's Maximum Principle and optimality conditions, optimal effort necessary to reduce the transmission rate of fowl pox in the poultry has been determined. Hence, it is possible to reduce to reduce the rate of transmission.
\end{abstract}

Key words: Optimal control, fowl pox, optimality condition, caseous deposits, mosquitoes remain infected, numerical solution setting, disease transmission

\section{INTRODUCTION}

Fowl pox, pox, or avian pox is a relatively slowspreading viral disease characterized by skin lesions or plagues in the pharynx. It is prevalent among chickens, turkey, pigeons, canaries, worldwide. Morbidity is $10-95 \%$ and mortality usually $0-50 \%$. Infection occurs through the skin abrasions and bites, or by the respiratory route.

The virus persists in the environment for months. The duration of the disease is about 14days on individual bird bases. The infected birds display some of the following symptoms: warty spreading eruption, scabs on comb and wattles caseous deposits in mouth throat and sometimes trachea, depression, poor growth and poor egg production. Because of its slow-spreading nature, it is possible to vaccinate to stop an outbreak. Flocks and individuals still unaffected may be vaccinated usually with chicken strain by wing web vaccinating method. If there is evidence of secondary bacterial infection, broad-spectrum, antibiotics may be of some benefit.

Fowl pox or avian pox is transmitted by direct contact between infected and susceptible birds or by mosquitoes. Virus-containing scabs also can be slough from infected birds and serve as a source of infection.
The virus can enter the blood stream through the eye, skin wound, or respiratory tracts. Mosquitoes become infected by feeding on birds with fowl pox in their blood stream. There is some evidence that the mosquitoes remain infected for life. Mosquitoes are the primary reservoir and spreaders of fowl pox on poultry ranges. Several species of mosquito can transmit fowl pox. Often mosquitoes winter-over in poultry houses, so outbreak can occur during winter and early spring.[ http://msucares.com/poultry/disease/disviral.htm]

Joshi (2002) in this study on Optimal control of HIV immunology model, illustrate the idea of optimal control on two types of disease model, the first is an epidemic model with two incidence forms, a percentage of the population are vaccinated to achive control of the disease. The percentage as a function of time is the control. While the second one illustrate drug treatment strategy in an immunology model.

Hee-Dae (2005), presented a work on the Application of optimal control theory to mathematical model of biological systems. In his study, he formulated a dynamic mathematical model for a vector-transmitted disease. He derived the condition necessary for optimality using lagrangian, optimal prevention and treatment effort was derived by formulating and analysing an optimal control

Corresponding Author: Udofia Ekere Sunday, Department of Mathematics and Computer Science Federal University of Technology Owerri, Imo State, Nigeria 
problem. It was shown that there are control effort for treatment of host and prevention of host-vector contact with minimal cost and side effect.

Ollerton (1989) in his study on the application of optimal control theory to diabetes mellitus, uses mathematical model and optimal control theory as grid search techniques and discrete segment approaches to derive a closed-loop insulin infusion algorithm for the metabolic control of hospitalised, non-insulin producing subjects.

Nanda et al. (2007), in their stduy on the Optimal Control of treatment in mathematical model of chronic mylogenous leukaemia described the interaction between naive $\mathrm{T}$ cell and leukemic cancer cells in a hypothetical patient with a system of ordinary differential equation. Optimal control theory was used to determine the treatment regimen that minimizes the cancer cell count and the deleterious effects of the drugs for a given patient. Both analytical and numerical solution setting were considered in determining the optimal control regimen under various assumptions.

Hattaf et al. (2009) in their study on Optimal Control of tuberculosis with exogenous reinfection, applied optimal control theory in the resulting ordinary differential equation to minimize the infectious group by the reduction of the contact between infection and the exposed individuals. Pontryagins Maximum Principle was used to characterize the optimal control; optimality system was derived and solved numerically.

Adekunle (2009), in their study on the application of optimal control to the epidemiology of tuberculosis transmission via the application of the Pontryagins Maximum of optimal control theory incorporated controls to a simple SEIR disease model of tuberculosis transmission dynamics to reduce the latently infected and actively infected individual populations.

Afshari et al. (2009), in their study on Determination of Nonlinear Optimal Feedback Law for Satellite Injection Problem Using Neighbouring Optimal Control investigated an optimal trajectory design of a nonlinear satellite injection problem for transfer to a final target orbit by minimizing the time. According to their investigation, this design was an exact solution to the nonlinear two-point boundary value problem which determined optimal control history as well as optimal state trajectories in the openloop form.

In this study, we present the mathematical model for application of optimal control to the epidemiology fowl pox transmission dynamics in poultry. Our goal is to determine optimal effort needed for the prevention and treatment of fowl pox in order to reduce incidence rate in poultry. As suggested by (Adekunle, 2009; Reluga et al.,
2010) that when virulence decreases with age the optimal behaviours for individual will minimize disease transmission. Here we seek for optimal effort necessary to reduce transmission rate of fowl pox in poultry.

\section{Fowl pox transmission model with chemoprophylaxis: \\ Assumptions:}

- Infected birds are treated

- The exposed (latent) birds are treated

- Recovered birds can join the susceptible population class

- Individuals die only by infection

The fowl pox transmission model divides the population of birds into the following sub-groups that are Susceptible birds (S), those exposed to fowl pox virus (E), those Infected with fowl pox and are displaying symptoms (I) and those that have been removed or Recovered from infection (R).

Parameters/symbols:

$\mathrm{S}=$ Susceptible population of birds at time $\mathrm{t}$

$\mathrm{E}=\operatorname{Exposed}($ Latent) population of birds at time $\mathrm{t}$

$\mathrm{I}=$ Infected population of birds at time $\mathrm{t}$

$\mathrm{R}=$ Removed population of birds at time $\mathrm{t}$

$\beta=$ Recruitment new birds that enter into the susceptible population

$\mathrm{A}=$ Transmission rate of infection

$\delta=$ The rate at which the susceptible join the exposed pop ulation class

$\mu=$ The rate at which the recovered birds join the susceptible population class

$r_{1}=$ The rate at which the exposed birds are taken care to avoid becoming infectious

$\mathrm{r}_{2}=$ The rate at which the infected birds are treated

$\mathrm{K}=$ Rate at which the exposed moves to infected population

$\mathrm{d}_{1}=$ Death rate due to infection

$\mathrm{d}_{2}=$ Death rate of the removed population

$\mathrm{R}=$ The rate at which the susceptible birds are bought in the poultry

$\delta=$ The rate at which susceptible birds are born into the poultry

The model:

Mode formulation: Our model describes the transmission dynamics of fowl pox infection based on two strains. Here we assume that birds which recover from one strain can become susceptible to the other strain. The susceptible population is increased by recruitment of birds either by birth $(\delta)$ or immigration 
(r) and the recovered bird $(\mu)$ that become susceptible. This population is reduced by infection and by natural death or emigration $(\delta)$ Using the above symbols/parameters and assumptions, we develop the model as follows Eq. 1-4:

$\frac{\mathrm{ds}}{\mathrm{dt}}=\beta \mathrm{s}-\mathrm{aSI}+\mu \mathrm{R}-\gamma \mathrm{S}$

$\frac{\mathrm{dE}}{\mathrm{dt}}=\gamma \mathrm{S}-\mathrm{kE}-\mathrm{r}_{1} \mathrm{E}$

$\frac{\mathrm{d} I}{\mathrm{dt}}=\mathrm{aSI}+\mathrm{kE}-\mathrm{d}_{1} \mathrm{I}-\mathrm{r}_{2} \mathrm{I}$

$\frac{\mathrm{dR}}{\mathrm{dt}}=\mathrm{r}_{2} \mathrm{I}+\mathrm{r}_{1} \mathrm{E}-\left(\mu+\mathrm{d}_{2}\right) \mathrm{R} \beta=\mathrm{r}+\delta$

Introducing the controls representing the treatment of the exposed and that of the infected, the model (1) (4) becomes Eq. 5-8:

$\frac{\mathrm{d} S}{\mathrm{dt}}=\beta \mathrm{S}-\mathrm{aSI}+\mu \mathrm{R}-\gamma \mathrm{S}$

$\frac{\mathrm{dE}}{\mathrm{dt}}=\gamma \mathrm{S}-\mathrm{kE}-\mu_{1} \mathrm{E}$

$\frac{\mathrm{dI}}{\mathrm{dt}}=\mathrm{aSI}+\mathrm{kE}-\mathrm{d}_{1} \mathrm{I}-\mathrm{u}_{2} \mathrm{I}$

$\frac{\mathrm{dR}}{\mathrm{dt}}=\mu_{2} \mathrm{I}+\mu_{1} \mathrm{E}-\left(\mu+\mathrm{d}_{2}\right) \mathrm{R} \beta=\mathrm{r}+\delta$

where, $\mathrm{S}(0), \mathrm{E}(0), \mathrm{I}(0), \mathrm{R}(0)$ are given, the definition of the model parameters are as given above. The control functions $\mathrm{u}_{1}(\mathrm{t})$ and $\mathrm{u}_{2}(\mathrm{t})$ are bounded lebesgue integrable functions. The control $\mathrm{u}_{1}(\mathrm{t})$ the time dependent effort on the treatment of latently infected birds practiced on the time interval $\left[0, \mathrm{t}_{\mathrm{f}}\right]$ to reduce the number of birds that may become fully infected. While $\mathrm{u}_{2}(\mathrm{t})$ is the effort on actively infected birds to increase the number of recovered birds

\section{MATERIALS AND METHODS}

Optimal control: Our optimal control problem involves that in which the number of birds with latent and active fowl pox infections and the cost of treatment controls $\mathrm{u}_{1}(\mathrm{t})$ and $\mathrm{u}_{2}(\mathrm{t})$ are minimized subject to the differential Eq. 5-8. This involves the number of birds with active and latent fowl pox respectively as well as the cost of applying chemoprophylaxis control $\mathrm{u}_{1}$ and treatment $\mathrm{u}_{2}$ in birds with fowl pox.
The objective function is defined as Eq. 9:

$J=\min _{\mathrm{u} 1, \mathrm{u} 2} \int_{0}^{t} f\left[K_{1} E+K_{2} I+C_{1} u_{1}^{2}+C_{2} u_{2}^{2}\right] d t$

where, $t_{\mathrm{f}}$ is the final time and the co-efficient $\mathrm{K}_{1}, \mathrm{~K}_{2}$, $\mathrm{C}_{1}, \mathrm{C}_{2}$ are balancing cost factors.

Our target is to minimize the objective functional defined in Eq. 9 by minimizing the number of the exposed and infectious classes. In order words, we seek to find the optimal pair $u_{1}^{*}$ and $u_{2}^{*}$ such that:

$\mathrm{J}\left(\mathrm{u}_{1}^{*}, \mathrm{u}_{2}^{*}\right)=\min \left[\mathrm{J}\left(\mathrm{u}_{1}, \mathrm{u}_{2}\right) / \mathrm{u}_{1}, \mathrm{u}_{2} \in \mathrm{U}\right]$

where, $\quad \mathrm{U}=\left\{\mathrm{u}_{1}(\mathrm{t}), \mathrm{u}_{2}(\mathrm{t}) / \mathrm{u}_{1}(\mathrm{t}), \mathrm{u}_{2}(\mathrm{t})\right.$ are measurable, $\left.a_{i} \leq\left(u_{1}(t) u_{2}(t)\right) \leq b_{i},=1,2, t^{\epsilon}\left[0, t_{f}\right]\right\}$ is the control set. Here $a_{i}$ and $b_{i}$ are constant in $[0,1]$.

Theorem 1: There exist optimal control $u_{2}^{*}, u_{2}^{*}$ and solutions $\mathrm{S}^{*}, \mathrm{E}^{*} \mathrm{I}^{*} \mathrm{R}^{*}$ of the corresponding state system (5-8) that minimizes $\mathrm{J}\left(\mathrm{u}_{1}, \mathrm{u}_{2}\right)$ over $\mathrm{U}$. Furthermore there exist $\lambda_{\mathrm{s}}, \lambda_{\mathrm{E}}, \lambda_{1}, \lambda_{\mathrm{R}}$ satisfying:

$$
\begin{aligned}
\frac{\mathrm{d} \lambda_{\mathrm{S}}}{\mathrm{dt}} & =\frac{\partial \mathrm{H}}{\partial \mathrm{S}} \\
\frac{\mathrm{d} \lambda_{\mathrm{E}}}{\mathrm{dt}} & =\frac{\partial \mathrm{H}}{\partial \mathrm{E}} \\
\frac{\mathrm{d} \lambda_{\mathrm{I}}}{\mathrm{dt}} & =\frac{\partial \mathrm{H}}{\partial \mathrm{I}} \\
\frac{\mathrm{d} \lambda_{\mathrm{R}}}{\mathrm{dt}} & =\frac{\partial \mathrm{H}}{\partial \mathrm{R}}
\end{aligned}
$$

With transversality conditions:

$$
\lambda_{\mathrm{i}}\left(\mathrm{t}_{\mathrm{f}}\right)=0, \mathrm{i}=\mathrm{S}, \mathrm{E}, \mathrm{I}, \mathrm{R}
$$

Moreover, the optimal control is given by:

$$
u_{1}^{*}=\min \left\{b_{1}, \max \left[a_{1}, \frac{E=\left(\lambda_{E}-\lambda_{R}\right)}{2 C_{1}}\right]\right\}
$$

And:

$$
\mathrm{u}_{1}^{*}=\min \left\{\mathrm{b}_{2}, \max \left[\mathrm{a}_{2}, \frac{\mathrm{I}\left(\lambda_{\mathrm{I}}-\lambda_{\mathrm{R}}\right)}{2 \mathrm{C}_{2}}\right]\right\}
$$

Proof: Corollary 4.1 of (Fleming and Rishel, 1975) gives the existence of an optimal control pair due to the convexity of the integrand of $J$ in (9) with respect to the control $\mathrm{u}_{1}$ and $\mathrm{u}_{2}$, a priori boundedness of the state solutions and the Lipschitz property of the state system with respect to the state variables. 
The differential equations governing the adjoint variables are obtained by differentiation of the Hamiltonian function, evaluated at the optimal control pair.

The adjoint system can be written as:

$$
\begin{aligned}
-\frac{\mathrm{d} \lambda_{\mathrm{S}}}{\mathrm{dt}} & =\frac{\partial \mathrm{H}}{\partial \mathrm{S}}, \lambda_{\mathrm{S}}\left(\mathrm{t}_{\mathrm{f}}\right)=0 \\
-\frac{\mathrm{d} \lambda_{\mathrm{E}}}{\mathrm{dt}} & =\frac{\partial \mathrm{H}}{\partial \mathrm{E}}, \lambda_{\mathrm{E}}\left(\mathrm{t}_{\mathrm{f}}\right)=0 \\
-\frac{\mathrm{d} \lambda_{\mathrm{I}}}{\mathrm{dt}} & =\frac{\partial \mathrm{H}}{\partial \mathrm{I}}, \lambda_{\mathrm{I}}\left(\mathrm{t}_{\mathrm{f}}\right)=0 \\
-\frac{\mathrm{d} \lambda_{\mathrm{R}}}{\mathrm{dt}} & =\frac{\partial \mathrm{H}}{\partial \mathrm{R}}, \lambda_{\mathrm{R}}\left(\mathrm{t}_{\mathrm{f}}\right)=0
\end{aligned}
$$

Where:

$$
\begin{gathered}
\frac{\partial \mathrm{H}}{\partial \mathrm{S}}=\lambda_{\mathrm{s}}(\beta-\mathrm{aI}-\gamma)+\lambda_{\mathrm{E}} \gamma+\mathrm{aI} \lambda_{1} \\
\frac{\partial \mathrm{H}}{\partial \mathrm{E}}=\mathrm{K}_{1}-\lambda_{\mathrm{E}}\left(\mathrm{k}+\mu_{1}\right)+\mathrm{k} \lambda_{1}+\lambda_{\mathrm{R}} \mathrm{u}_{1} \\
\frac{\partial \mathrm{H}}{\partial \mathrm{I}}=\mathrm{k}_{2}-\lambda_{\mathrm{s}}(\mathrm{aI})+\lambda_{\mathrm{I}}\left(\mathrm{aI}-\mathrm{u}_{2}\right)+\lambda_{\mathrm{R}} \mathrm{u}_{2} \\
\frac{\partial \mathrm{H}}{\partial \mathrm{S}}=\lambda_{\mathrm{s}} \mu-\lambda_{\mathrm{R}}\left(\mu+\mathrm{d}_{2}\right)
\end{gathered}
$$

Therefore:

$$
\begin{gathered}
\frac{\mathrm{d} \lambda_{\mathrm{s}}}{\mathrm{dt}}=\lambda_{\mathrm{s}}(\mathrm{aI}+\gamma-\beta)-\lambda_{\mathrm{E}} \gamma-\mathrm{aI} \lambda_{1} \\
\frac{\mathrm{d} \lambda_{\mathrm{E}}}{\mathrm{dt}}=\lambda_{\mathrm{E}}\left(\mathrm{k}+\mu_{1}\right)-\mathrm{k}_{1} \mathrm{k} \lambda_{\mathrm{I}}-\lambda_{\mathrm{R}} \mu_{1} \\
\frac{\mathrm{d} \lambda_{\mathrm{I}}}{\mathrm{dt}}=\lambda_{\mathrm{S}}(\mathrm{aI})-\mathrm{k}_{2}-\lambda_{\mathrm{I}}\left(\mathrm{aI}-\mathrm{u}_{2}\right)-\lambda_{\mathrm{R}} \mathrm{u}_{2}
\end{gathered}
$$

We shall use the optimality conditions:

$$
\frac{\partial \mathrm{H}}{\partial \mathrm{u}_{\mathrm{i}}}=0
$$

where, $\mathrm{i}=1,2$ :

$$
\frac{\partial \mathrm{H}}{\partial \mathrm{u}_{1}}=0 \frac{\partial \mathrm{H}}{\partial \mathrm{u}_{2}}=0
$$

And:

$$
\begin{gathered}
\frac{\partial \mathrm{H}}{\partial \mathrm{u}_{1}}=2 \mathrm{C}_{2} \mathrm{u}_{2}^{*}-\lambda_{1} \mathrm{I}+\lambda_{\mathrm{R}} \mathrm{I}=0 \\
\frac{\partial \mathrm{H}}{\partial \mathrm{u}_{1}}=\frac{\mathrm{d} \lambda_{\mathrm{I}}}{\mathrm{dt}}=\lambda_{\mathrm{S}}(\mathrm{aI})-\mathrm{k}_{2}-\lambda_{\mathrm{I}}\left(\mathrm{aI}-\mathrm{u}_{2}\right)-\lambda_{\mathrm{R}} \mathrm{u}_{2}
\end{gathered}
$$

Hence, we obtain:

$$
\begin{aligned}
& u_{1}^{*}=\frac{E\left(\lambda_{E}-\lambda_{R}\right)}{2 C_{1}} \\
& u_{2}^{*}=\frac{1\left(\lambda_{1}-\lambda_{R}\right)}{2 C_{2}}
\end{aligned}
$$

Then by standard control argument involving the bounds on the controls, we conclude for control $\mathrm{u}_{1}$ and $\mathrm{u}_{2}$ :

$$
u_{1}^{*}=\min \left\{b_{1}, \frac{E\left(\lambda_{E}-\lambda_{R}\right)}{2 C_{1}}\right\}
$$

And:

$$
u_{2}^{*}=\min \left\{b_{2}, \frac{1\left(\lambda_{1}-\lambda_{R}\right)}{2 C_{2}}\right\}
$$

\section{RESULTS}

Analysis of optimal control: Using Pontryagin's Maximum principle, we formulate the Hamiltonian from the cost functional (9) and the governing dynamic Eq. 5-8 to obtain the optimality conditions Eq. 10:

$$
\begin{aligned}
& \mathrm{H}=\mathrm{k}_{1} \mathrm{E}+\mathrm{K}_{2} \mathrm{I}+\mathrm{C}_{1} \mathrm{u}_{1}^{2}+\mathrm{C}_{2} \mathrm{u}_{2}^{2} \\
& +\lambda \mathrm{s}(\beta \mathrm{S}-\mathrm{aSI}+\mu \mathrm{R}-\gamma \mathrm{S}) \\
& +\lambda_{\mathrm{E}}\left(\gamma \mathrm{S}-\mathrm{kE}-\mathrm{u}_{1} \mathrm{E}\right)+\lambda_{1}\left(\mathrm{aSI}+\mathrm{kE}-\mathrm{d}_{1} \mathrm{I}-\mu_{2} \mathrm{I}\right) \\
& +\lambda_{\mathrm{R}}\left(\mathrm{u}_{2} \mathrm{I}+\mathrm{u}_{1} \mathrm{E}-\left(\mu+\mathrm{d}_{2}\right) \mathrm{R}\right)
\end{aligned}
$$

where, the $\lambda_{\mathrm{s}}, \lambda_{\mathrm{E}}, \lambda_{1}, \lambda_{\mathrm{R}}$ are the associated adjoint for the epidemiological states $S, E, I, R$ The system of equation is found by taking the appropriate partial derivative of the Hamiltonian with respect to the associated state variable. Using the optimality condition and by standard control argument involving the bounds on the controls, we obtain the solution of the optimal control problem as control:

$$
u_{1}^{*}=\min \left\{b_{1}, \frac{E\left(\lambda_{E}-\lambda_{R}\right)}{2 C_{1}}\right\}
$$

And:

$$
u_{2}^{*}=\min \left\{b_{2}, \frac{1\left(\lambda_{1}-\lambda_{R}\right)}{2 C_{2}}\right\}
$$

\section{DISCUSSION}

Herd immunity describes a form of immunity that occurs when the vaccination of a significant portion of 
a population (or herd) provides a measure of protection for individuals who have not developed immunity John and Samuel (2000). Herd immunity theory proposes that, in contagious diseases that are transmitted from individual to individual, chains of infection are likely to be disrupted when large numbers of a population are immune or less susceptible to the disease. The greater the proportion of individuals who are resistant, the smaller the probability that a susceptible individual will come into contact with an infectious individual. The effort of applying chemoprophylaxis control $\mathrm{u}_{1}$ and treatment control $\mathrm{u}_{2}$ in birds with fowl pox is aimed toward reducing the number of birds that are infected with fowl pox. This will enable us achieve 'herd immunity' hence controling the rate of transmission of infection.

\section{CONCLUSION}

In this study, we present the mathematical model of the transmission dynamics of fowl pox infection in poultry. It describes the interaction between the susceptible and the infected birds which results in a system of ordinary differential equation. Introducing the control which represents the effort in applying chemoprophylaxis control $\mathrm{u}_{1}$ and treatment control $\mathrm{u}_{2}$ in birds with fowl pox, the system becomes a system of ordinary differential equation with control. Analysing the model using Pontryagin's Maximum Principle and optimality conditions, optimal effort necessary to reduce the transmission rate of fowl pox in the poultry has been determined to be $u_{1}^{*}=\frac{E\left(\lambda_{E}-\lambda_{R}\right)}{2 C_{1}} \quad$ and $u_{2}^{*}=\frac{I\left(\lambda_{I}-\lambda_{R}\right)}{2 C_{2}}$ this effort is partially aimed at achieving the herd immunity of the infection.

\section{REFERENCES}

Adekunle, A.I., 2009. Application of optimal control to the epidemiology of tuberculosis transmission, department of mathematical sciences. Federal University of Technology, Akure.
Afshari, H.H., A.B. Novinzadeh and J. Roshanian, 2009. Determination of nonlinear optimal feedback law for satellite injection problem using neighboring optimal control. Am. J. Applied Sci., 6: 430-438. DOI: 10.3844/ajassp.2009.430.438

Fleming, W.H. and R.W. Rishel, 1975. Deterministic and Stochastic Optimal Control. 1st Edn., SpringerVerlag, New York, ISBN-10: 0387901558, pp: 222.

Hattaf, K., M. Rachik, S. Saadi, S. Tabit and N. Yousfi, 2009. Optimal control of tuberculosis with exogenous reinfection. J. Applied Mathe. Sci., 3: 231-240.

Hee-Dae, K., 2005. Application of optimal control theory to mathematical model of biological systems. Inha University, Incheon, Korea.

John, T.J. and R. Samuel, 2000. Herd immunity and herd effect: New insights and definitions. Eur. J. Epidemiol., 16: 601-606. PMID: 11078115

Joshi, H.R., 2002. Optimal control of an HIV immunology model. J. Optimal Control Application Methods, 23: 199-213. DOI: 10.1002/oca.710

Nanda, S., H. Moore and S. Lenhart, 2007. Optimal control of treatment in a mathematical model of chronic myelogenous leukemia. J. Math. Biosci., 210: 143-156. PMID: 17599363

Ollerton, R.L., 1989. Application of optimal control theory to diabetes mellitus. Int. J. Control, 50: 2503-2523. DOI: 10.1080/00207178908953512

Reluga, T.C., J. Medlock, E. Poolman and A.P. Galvani, 2010. Optimal timing of disease transmission in an age-structured population. Bull. Math Biol., 69: 2711-2722. 